\title{
Extracellular Vesicles: Biomarkers, Therapeutics, and Vehicles in the Visual System
}

\author{
Yolandi van der Merwe $\mathrm{e}^{1,2,3} \cdot$ Michael B. Steketee $\mathrm{e}^{2,3,4}$
}

Published online: 6 October 2017

(C) Springer Science+Business Media, LLC 2017

\begin{abstract}
Purpose We discuss recent advances in extracellular vesicle (EV) technology as biomarkers, therapeutics, and drug delivery vehicles in the visual system with an emphasis on the retina.

Recent Findings Retinal cell-type specific EVs can be detected in the blood and in the aqueous humor and EV miRNA cargoes can be used diagnostically to predict retinal disease progression. Studies have now shown EVs can deliver bioactive miRNA and AAV cargoes to the inner retinal cell layers and, in some models, improve retinal ganglion cell (RGC) survival and axon regeneration.

Summary EV molecular profiles and cargoes are attractive biomarkers for retinal and optic nerve disease and trauma and EVs offer a safe and tunable platform for delivering therapies to ocular tissues. However, EVs are heterogeneous by nature with variable lipid membranes, cargoes, and biologic effects, warranting stringent characterization to understand
\end{abstract}

This article is part of the Topical Collection on Regenerative Medicine in Ophthalmology

Michael B. Steketee

SteketeeM@UPMC.edu

1 Department of Bioengineering, University of Pittsburgh, Pittsburgh, PA 15260, USA

2 Department of Ophthalmology, University of Pittsburgh, Pittsburgh, PA 15216, USA

3 McGowan Institute for Regenerative Medicine, University of Pittsburgh, 450 Technology Drive, Suite 300, Pittsburgh, PA 15219, USA

4 Center for Neuroscience, University of Pittsburgh, Pittsburgh, PA 15261, USA how heterogeneous EV populations modulate positive tissue remodeling.

Keywords Extracellular vesicles $\cdot$ Exosomes $\cdot$ Biomarkers · Retina $\cdot$ Optic nerve $\cdot$ Retinal ganglion cell $\cdot$ miRNA

\section{Introduction}

Extracellular vesicles (EVs) are increasingly appreciated as important factors regulating intercellular communication in the visual system and are now being explored as biomarkers, therapeutics, and drug delivery vehicles. EVs comprise several populations of sub-micron sized vesicles, including exosomes, ectosomes, oncosomes, shed vesicles or microvesicles, apoptotic bodies, and matrix-bound nanovesicles (MBVs). Though the EV nomenclature is developing, EVs are generally classified by their biogenic origin and molecular profiles into four main groups: exosomes, microvesicles, apoptotic bodies [1], and the recently described MBVs [2]. Each group is characterized by distinct sets of lipids, proteins, and nucleic acids. However, EVs are heterogeneous even within subgroups and EV subgroup localizations are wide spread within tissues, organs, and body fluids and thus overlap significantly. EVs are now recognized widely as evolutionarily conserved intercellular communication vehicles that regulate cellular proliferation, migration, organization, and phenotypes during development, maintenance and function, injury and disease, and in aging [3]. Hence, EV research has increased dramatically in recent years on EV pathophysiology and the use of EVs as biomarkers and potential therapeutics. Here, we discuss recent EV findings in the visual system, using EVs to diagnose or to treat injury or disease in the visual system, specifically retinal and optic 
nerve diseases or trauma, and logical next steps required to advance $\mathrm{EV}$ technology responsibly to the clinic.

\section{Extracellular Vesicle Overview}

EVs are lipid-bound vesicles ranging in size from 10 to $1000 \mathrm{~nm}$. EVs are secreted from virtually all cell types and are found in all body fluids and the extracellular matrix. EVs are secreted via numerous mechanisms, including direct budding or shedding from the parent cell, release from multivesicular bodies fusing with the plasma membrane, exocytosis, or via apoptotic cell body release. Though, in some cases, EV membranes may resemble the parent membrane compositionally as in budding or shedding microvesicles, EV lipid membranes are generally distinct from the parent plasma membrane and these distinctions play important roles in modulating the biophysical properties of EVs both before and after secretion. Prior to secretion, unique lipid compositions influence interactions with other lipids and proteins, like recruiting effector molecules necessary for EV sorting, trafficking, and micro-domain localization, and the timing, location, and rate of secretion, among other factors. After secretion, lipid and membrane composition regulates the rate and timing of EV transport within tissues and fluids, cellular recognition, location and method of uptake, and ultimately functional impact. EV membranes are differentially enriched in various lipid modifiers, like enzymatic phospholipases, and lipids like cholesterol and sphingomyelin, which are hypothesized to increase EV lipid packing density, enhancing EV stability during transport within body fluids and increasing cellular uptake specificity [4]. Moreover, EVs can also carry bioactive releasable lipids like prostaglandins and arachidonic acid [5, 6].

\section{EV Surface and Cargo Properties and Signaling}

Regardless of EV type, EV properties generally reflect the phenotype of the parent cell; surface markers, cargoes, secretion rate, and location reflect the parent cell's physiology [7•]. In addition to lipids, EV surface molecules include carbohydrates, and proteins that regulate EV secretion temporally and spatially, inter- and intra-cellular transport within tissue and body fluids, and cellular targeting. EVs are equipped to signal via multiple direct contact mechanisms, including cell surface receptor activation. Cells can internalize EVs by different mechanisms, including clathrin-dependent and clathrinindependent endocytoses, phagocytosis, macropinocytosis, and fusion mediated by lipid rafts [8]. Surface proteins, lipids, and carbohydrates direct cellular targeting as well as the mechanism and the location of internalization. Both the mechanism and the location influence the biological effects of EVs and their cargoes, important considerations for therapeutic development. Like surface markers, EV cargoes are variable, but generally include proteins and nucleic acids, particularly miRNAs, that reflect the phenotype and the release site from the parent cell, particularly important in highly polarized cells, like neurons, vascular endothelial cells, and the various cells making up the blood-brain barrier, including retinal pigment epithelial (RPE) cells that make up the outer blood retinal barrier (BRB) and Müller glia and astrocytes that make up the inner BRB. Importantly, EVs, regardless of the vesicle subtype or cellular source, are heterogeneous populations with distinct surface markers, cargoes, and biologic effects (Fig. 1) [9•]. For example, a recent study showed EVs from different stem cell populations can have opposite effects on neurite growth from different primary neurons [10]. Thus, both preclinically and clinically increased stringencies are needed to establish guidelines for the characterization of surface markers and cargoes, and rational delivery strategies must be developed to effectively advance EV-based therapies clinically.

\section{EVs as Biomarkers in the Visual System}

EV molecular profiles are increasingly recognized as potential biomarkers for diseases, including cancers and neurodegenerative diseases in the CNS. In spinal cord injury (SCI), changes in exosomal miRNAs reflect many of the barriers to CNS regeneration. For example, SCI can differentially regulate exosomal miRNAs that modulate calcium signaling, synaptic function, axon guidance [11-13], axon degeneration, inhibitory molecule expression, and scar tissue formation [14], suggesting engineered EV combinations may be useful in counteracting or modulating the signaling pathways underlying the default healing response in the CNS. Though EV biology in the visual system is less developed, a number of recent studies indicate EVs, like exosomes and microvesicles, and their cargoes, particularly miRNAs and inflammatory proteins, may be used to detect, monitor, and prognosticate retinal and possibly optic nerve trauma and disease.

For example, rhegmatogenous retinal detachment (RD) is the most common type of $\mathrm{RD}$ and proliferative vitreoretinopathy (PVR) is the most common disease that develops from RD. PVR can complicate photoreceptor reattachment to the RPE by causing malapposition and lost visual acuity or even photoreceptor death and vision loss. However, the underlying mechanical and inflammatory mechanisms are only partially understood. A recent study showed that EVs derived from photoreceptors, peanut agglutinin or phosphatidylserine-positive, were higher in the aqueous humor $(\mathrm{AH})$ of patients after rhegmatogenous RD [15•]. The photoreceptor-specific EVs were higher depending on the duration of the $\mathrm{RD}$ at the time of surgery and closely correlated to soluble pro-inflammatory factors, like MCP-1, a proinflammatory cytokine involved in photoreceptor apoptosis. 


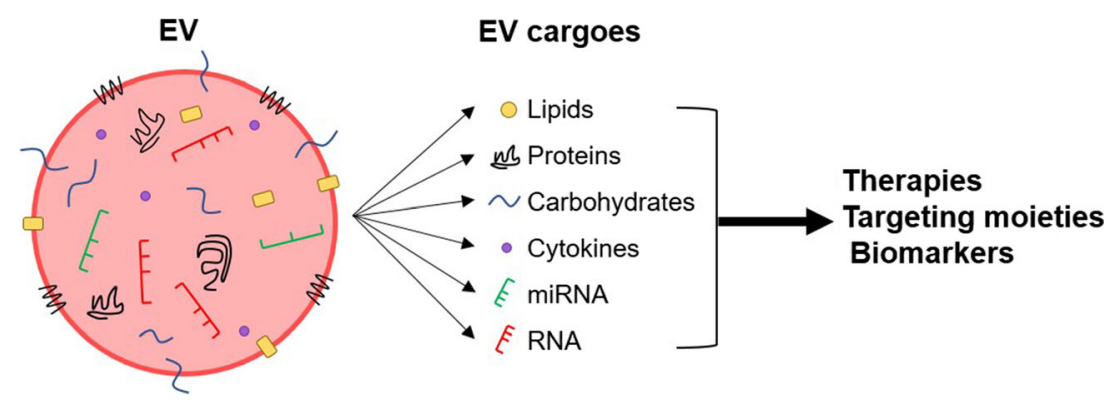

Fig. 1 EVs are composed of various molecules capable of cellular signaling that may also be used therapeutically or for biomarker analysis. EVs are secreted from cells throughout the body and carry lipid, protein, carbohydrate, cytokine, miRNA, and RNA cargoes. EV

Thus, retinal cell-type specific EVs can be detected after RD, and $\mathrm{EV}$ concentrations are predictive of the RD duration and pro-inflammatory factors known to induce photoreceptor apoptosis, supporting the notion that analysis of vitreal EVs may aid in the prognosis of RD surgery and visual recovery.

MiRNA cargoes within EVs can serve as biomarkers for disease, not only in the body fluid affected by disease, but systemically in the blood levels as well. MiR146a and miR26a were upregulated in the vitreous humor of uveal melanoma patients [16], and follow-up studies revealed miR146a was also upregulated in blood serum exosomes of uveal melanoma patients [17]. Additionally, systemic miRNAs are being investigated as biomarkers for malignant gliomas [18]. In brain tissue, miRNA expression is up- or downregulated for numerous miRNA with glioma onset and progression, and preliminary clinical data suggests circulating miRNA may serve as a biomarker for glioma diagnosis and prognosis.

EV detection and analysis may also eventually predict predisposition or progression in glaucomatous eyes. The causes of glaucoma are unknown; however, one factor commonly associated with glaucoma is an increase in intraocular pressure (IOP). Even though increased IOP is associated with the disease and often used as a predictor for glaucoma, IOP increase is not present in all glaucoma cases, and frequently IOP increase is only observed after significant visual field loss. Therefore, a need exists for a method to diagnose early onset glaucoma while also determining the underlying cause of the disease. In glaucoma patients, specific subsets of miRNAs are upregulated and downregulated, respectively [19], suggesting there is direct or indirect miRNA signaling between retinal ganglion cells (RGCs) and the vitreous, and additional studies indicate the majority of miRNAs in the $\mathrm{AH}$ are found within EVs [20]. Recent studies showed that non-pigmented ciliary epithelium cell (NPCE)-derived EVs accumulate in trabecular meshwork cells and effect Wnt signaling, a pathway involved in IOP regulation [21•]. Overall, these studies indicate EVs and their cargoes can serve as biomarkers for glaucoma, potentially serving as an early diagnostic marker, and may also contribute directly to the progression of the disease. surface proteins, lipids, and carbohydrates direct cellular targeting, can activate cell surface signaling, and influence the mechanism and location of internalization

\section{Developing EV Technology Clinically Requires Stringent, Rigorous Methodologies}

The number of studies on ocular EVs has rapidly expanded recently and consequently so has our understanding of EV biology in ocular disease and trauma and, inevitably, so has the number of proposed EV-based therapeutics with many studies showing promise in experimental models. However, as noted above, stringent purification and rigorous characterization are required to develop effective biomarkers and clinical therapies. For example, numerous cells secrete EVs into the $\mathrm{AH}$ or blood, and numerous molecules, particularly miRNAs, have been shown to change in response to ocular disease or injury. However, due to experimental methods and design, whether miRNAs in the $\mathrm{AH}$ are within cells, different EV populations, or within protein/RNA complexes is largely unknown. Moreover, we know virtually nothing about the cellular origins of EVs in the AH. To accurately capture and analyze cell-specific EV populations secreted from ocular tissues, within the AH or within the blood, cell-specific EV surface markers and cargo profiles must be identified. To date, a few cell-specific markers have been identified, as demonstrated for photoreceptors [15•]. However, we currently lack markers for most retinal cells, including RGCs and Müller glia. Moreover, some EVs may be tissue or compartment specific, which has been shown for some miRNAs in the anterior chamber [22]. Thus, minimally invasive and highly sensitive diagnostic tools must be developed specifically for ocular tissues.

In the future, EVs could be used in routine clinical screenings to measure the health of ocular tissues, including the retina and the optic nerve, and improve trauma and disease detection and treatment. However, to achieve clinical reliability, set purification, analytical standards, and technological advances in EV capture and analysis are required. EV purification requires stringent methodologies since $\mathrm{EV}$ profiles are affected by changes in the method of preparation, variations in cell cultures, and even batch-to-batch variability. Standardized purification methods have been established $[23,24]$ and 
refined [7•]; however, many investigators have yet to adopt these standards. EV purification kits are often used for convenience. However, preparations from these kits are often contaminated with other vesicles, non-exosomal proteins, and nucleic acids, which can alter bioactivity and experimental results. Progress is also being made in developing nanoscale devices for capturing even single EVs [25]. Developing such tools that can capture low level exosomes in blood for instance is more patient friendly than probing ocular tissues and fluids. The ability to detect cell-type specific EVs will more rapidly advance our knowledge of EV biology in normal and pathogenic conditions and logically accelerate therapeutic developments. Developing standards for classifying EVs is also underway with the development of the ExoCarta database [26•]. Key to all of the above is developing proper analytic methods that can characterize EV rigorously.

However, to develop more refined EV capture methods requires more refined analytic methods. For example, exosomes released from the RPE have been hypothesized to play important roles in AMD pathogenesis. AMD is one of the leading causes of vision loss in people over age 60 and can present as either the "dry" form characterized by the presence of drusen deposits in the macula or as the "wet" form characterized by choroidal neovascularization. Changes in exosomal properties have been reported in the AH of AMD patients [27], and studies suggest exosomes may play a role in the development of choroidal neovascularization since exosomal markers are upregulated in aged RPE. Both drusen and oxidative stress can increase exosome secretion from RPE cells, suggesting exosomes may also play a role in dry AMD. In addition to metabolic support and maintenance of photoreceptors, the RPE plays several other roles critical to retinal health and function by forming the outer BRB in the eye. The inherent polarization of the BRB logically suggests RPE cell signaling, including EVs, must also be polarized to maintain normal retinal function and loss of such polarity would be expected to lead to RPE dysfunction as observed in aged RPE and AMD. Though methods have been developed to isolate exosomes clinically from AMD patient serum [28], further biomarker development is required to develop specific biomarkers to capture and analyze exosomes from normal or diseased RPE cells.

A recent study by Klingeborn et al. (2017) is a step in this direction by analyzing the proteome in EVs secreted by polarized RPE monolayers. Using mass spectrometry and protein correlation profiling, exosomes were analyzed from polarized, primary RPE monolayers grown on membranes in serum-free trans-well cultures. This approach allowed EVs to be analyzed from either the apical or the basolateral compartments. Consistent with the RPE's relationship with the retinal (apical) and systemic circulation (basolateral) in vivo, the authors demonstrated in vitro that epithelial polarity regulates directional exosome release; both the number of exosomes and their proteomes were polarized and consistent with RPE function and known protein localizations in vivo. Out of the 631 identified proteins, $47 \%$ were unique to apical exosomes and $15 \%$ unique to basolateral exosomes, with $37 \%$ found in both exosomal compartments [7•]. Though these results need to be confirmed in vivo, the demonstration of polarity-driven exosome secretion from polarized epithelial cells advances our understanding of EV biology and may be relevant to dysfunction in other epithelial tissues, like vascular retinopathies. Moreover, the differences in apical and basolateral exosomal proteomes advance the number of potential RPE-specific exosome markers in a polarity-dependent manner. Thus, not only changes in exosomal proteins, but also in their polarity, could be used to potentially monitor RPE function as part of preventative care, in the diagnosis and monitoring RPE dysfunction at an early disease stage, and/or monitor the effects of therapeutics on RPE function.

\section{EVs as Ocular Therapeutics}

Compared to cell-based therapies, EV-based therapies are attractive alternatives. In particular, stem cell (SC)-derived EVs have received most of the attention since several studies have shown SC EVs can recapitulate many of the positive therapeutic effects of the parent stem cell on immunomodulation and tissue remodeling without the negative inflammatory and neoplastic concerns associated with using SCs. EVs are small and, depending on their surface properties, can cross many biological barriers including the BBB. As expanded below, biologically produced EVs are easily customized using mainstream molecular and genetic techniques, including bar coding with nucleic acid or other molecular tags for tracking. Purified EVs are easily stored and loaded with small molecules, proteins, and nucleic acids, like miRNAs or even viral vectors. EVs or combinations of EVs can target and deliver diverse biomolecular cargoes to modulate distinct cellular populations, like immune cells, glia, and neurons, making them attractive developing combinatorial therapeutics to treat CNS and ocular injuries and diseases clinically. Though clinical studies are in early development phases, this idea is supported by pre-clinical rodent studies reporting positive tissue remodeling and increased functional recovery in a stroke model [29].

Recent pre-clinical studies have shown EVs can also positively modulate injury responses in the retina in optic neuropathy and neurovascular disease animal models. Damage to the optic nerve in ischemic optic neuropathies (ION) and traumatic optic neuropathies (TON) often leads to progressive and permanent vision loss. Though surgery or corticosteroids may benefit some patients, lost vision is likely permanent due to progressive RGC axon degeneration and death. After acute optic nerve ischemia in rat, a recent study by Mead et al. 
(2017) showed that intravitreally injected EVs, derived from bone marrow derived stem cells (BMSCs), successfully delivered their cargoes to the inner retinal layers and both Müller glia and RGCs. In BMSC EV-treated animals, RGC survival and axon regeneration increased and RGC axonal loss and RGC dysfunction decreased. The authors concluded these effects relied on exosomal miRNA, since the positive therapeutic effects on RGCs were diminished by knocking down the miRNA effector molecule Argonaute-2 [30•]. Fibroblast EVs have been reported to increase RGC survival and growth after acute optic nerve ischemia via established axon growth regulatory pathways involving mTOR activation by GSK3 $\beta$ and TSC2 [31•]. The ability of EVs to deliver bioactive nucleic acids to multiple layers in the inner retina suggests cell-free EV therapies may benefit other traumatic or neurodegenerative ocular diseases.

In support of this notion, EVs were recently shown to ameliorate ischemia and neovascularization in a vascular neuropathy model, possibly due to the positive immunomodulatory effects of SC EVs. Clinically, neovascular disease can lead to vision loss in several retinopathies, including retinopathy of prematurity, ischemic retinal vein occlusions, wet age-related macular degeneration, and proliferative diabetic retinopathy. Typical treatments include anti-VEGF drugs, thermal laser treatment, or photodynamic therapy. However, each is limited, requires prolonged or multiple treatments, and increases vision loss in some cases. Moisseiev et al. (2017) showed that intravitreally injected EVs from human MSCs significantly reduced retinal ischemia and neovascularization in a murine model of oxygen-induced retinopathy (OIR), the most widely used model for vascular pathology and abnormal angiogenesis, without immunosuppression [32]. MSC-derived EVs can modulate microglial-induced inflammation [33] which can in turn reduce astrocyte proliferation and activation [34]. MSCderived EVs has also been reported to reduce monocyte chemotactic protein (MCP)-1 expression and injury, apoptosis, and inflammatory responses in a mouse model of laser induced retinopathy [35 $]$. These initial findings are encouraging and motivate EV engineering to optimize or customize biologically produced EVs to deliver specific cargoes to specific cellular populations.

The above studies indicate good retinal biocompatibility with EVs derived from different cell sources and used in different experimental rodent models. EVs have several other desirable experimental advantages that will likely speed our knowledge on EV biology and the development of EV-based therapies. Unlike cells, EVs do not replicate, change phenotype, or actively migrate from the application site and thus can be manipulated and delivered with more precision. Some EVs, like exosomes, can cross the BBB, including the BRB, without initiating a pro-inflammatory immune response. EVs are easily derived from virtually any cellular source in vitro, including stem cells, immune system cells, glia, and tissue- specific cells, and are easily generated, purified, and stored for prolonged periods of time. Since EVs reflect the phenotype of the parent cell, EVs from cells cultured under controlled and varied physiological conditions are valuable in vitro and in vivo experimental tools. For example, identifying EV surface markers, cargoes, and cell-specific effects on polarized cells like microglia or astrocytes will provide new insight into microglia-mediated astrocyte A1 activation [34] and reveal new approaches to minimizing secondary trauma and scar tissue formation in the injured CNS [36].

\section{Engineered EVs as Therapies and Drug Delivery Vehicles}

Engineered EVs designed to deliver specific cargoes to specific cellular populations in a controllable manner offer the most attractive EV-based therapeutic for clinical use. Engineered EVs may be superior to single-molecule drugs, biologics, whole cells, and synthetic liposome or nanoparticle formulations because of the ease of bioengineering with multiple factors while retaining superior biocompatibility and biostability and posing fewer risks for abnormal differentiation or neoplastic transformation. [3]. EVs can be engineered to incorporate proteins, small molecules, and nucleic acids. Proteins can be concentrated in the lumen of EVs by fusing its partner with tetraspanin CD63, and miRNAs can be incorporated into EVS by fusing Ago protein with CD63. Additionally, Poly A, a binding protein that binds mature mRNAs, can selectively recruit mRNAs to EVs. IncRNAs can be enriched in EVs by fusing motifs from polycomb repressive complex 2 (EZH1 and EZH2) with tetraspanins. Using knowledge gained from biologically derived EVs, bioengineers are making progress toward developing homogeneous EV populations that can be engineered to target defined cargoes specific to certain cellular populations. While direct EV delivery is possible in most tissues throughout the body, retinal drug and therapeutic nucleic acid delivery are complicated by the BRB. Some EVs, like MSC exosomes, appear to readily cross the inner limiting membrane and can deliver nucleic acid cargoes to multiple retinal layers. Since EVs reflect the cargoes and state of the parent cell, treating cells in vitro with specific cytokines, growth factors, and small molecules to stimulate the production and release of EVs with desirable cargoes for therapeutic use is possible.

For example, a recent study by Wassmer et al. (2017) showed EV associated adeno-associated virus (Exo-AAV) can be delivered to the retina, and this EV-associated delivery mechanism outperformed standard AAV delivery with respect to green fluorescent protein (GFP) delivery to the retina [37]. GFP was packaged into Exo-AAV and conventional AAV vectors and delivered to the retina of adult mice via intravitreal injection. GFP expression was monitored by fundus imaging, 
histology, and qRT-PCR 4 weeks post-injection. Target cells for delivery were RGCs, bipolar cells, Müller cells, and photoreceptors, and results showed the Exo-AAV penetrated the inner nuclear, outer plexiform, and outer nuclear layers which was deeper than the AAV vector alone. The study indicates Exo-AAV serves as a robust gene delivery tool in the mouse retina, and the simplicity of production and isolation should make the Exo-AAV a widely applicable tool to ocular research.

Finally, MSCs have been used clinically to treat patients with graft versus host disease (GvHD), and a recent study showed that MSC-derived EVs are able to significantly decrease the symptoms of GvHD shortly after the onset of treatment [38]. MSC-derived EVs induced similar antiinflammatory effects as MSC, suggesting the EVs released from MSC, more so than the MSC themselves, may be responsible for the altered immune response after MSC therapy administration. Cell transplantation is fraught with caveats [36]; therefore, EV administration may be a safer and more reliable alternative to cell transplantation.

Funding Information We gratefully acknowledge funding from the Department of Defense office of the Congressionally Directed Medical Research Programs and the Clinical and Rehabilitative Medicine Research Program (MBS, W81XWH-15-1-0026), National Institutes of Health CORE Grant P30 EY008098, and Eye and Ear Foundation of Pittsburgh, PA, Unrestricted Grant from Research to Prevent Blindness, New York, NY.

\section{Compliance with Ethical Standards}

Conflict of Interest The authors declare that they have no conflict of interest.

Human and Animal Rights and Informed Consent This article does not contain any studies with human or animal subjects performed by any of the authors.

\section{References}

Papers of particular interest, published recently, have been highlighted as:

\section{- Of importance}

1. Kowal J, et al. Proteomic comparison defines novel markers to characterize heterogeneous populations of extracellular vesicle subtypes. Proc Natl Acad Sci USA. 2016;113(8):E968-77.

2. Huleihel L, et al. Matrix-bound nanovesicles within ECM bioscaffolds. Sci Adv. 2016;2(6):e1600502.

3. Riazifar M, et al. Stem cell extracellular vesicles: extended messages of regeneration. Annu Rev Pharmacol Toxicol. 2017;57:125-54.

4. Yoon YJ, Kim OY, Gho YS. Extracellular vesicles as emerging intercellular communicasomes. BMB Rep. 2014;47(10):531-9.

5. Koniusz S, et al. Extracellular vesicles in physiology, pathology, and therapy of the immune and central nervous system, with focus on extracellular vesicles derived from mesenchymal stem cells as therapeutic tools. Front Cell Neurosci. 2016;10:109.

6. Subra C, et al. Exosomes account for vesicle-mediated transcellular transport of activatable phospholipases and prostaglandins. J Lipid Res. 2010;51(8):2105-20.

7. Klingeborn M, et al. Directional exosome proteomes reflect polarity-specific functions in retinal pigmented epithelium monolayers. Sci Rep. 2017;7(1):4901. Klingeborn et al. demonstrated epithelial polarity regulates directional exosome release; both the number of exosomes and their proteomes were polarized with respect to the apical and basolateral RPE compartments.

8. Mulcahy LA, Pink RC, Carter DR. Routes and mechanisms of extracellular vesicle uptake. J Extracell Vesicles. 2014;3.

9. Willms E, et al. Cells release subpopulations of exosomes with distinct molecular and biological properties. Sci Rep. 2016;6: 22519. Regardless of cellular source, EVs are heterogeneous populations with distinct surface markers, cargoes, and biologic effects.

10. Lopez-Verrilli MA, et al. Mesenchymal stem cell-derived exosomes from different sources selectively promote neuritic outgrowth. Neuroscience. 2016;320:129-39.

11. Chandran R, et al. Differential expression of microRNAs in the brains of mice subjected to increasing grade of mild traumatic brain injury. Brain Inj. 2016:1-14.

12. Liu Y, et al. Bioinformatics analysis of microRNA time-course expression in brown rat (Rattus norvegicus): spinal cord injury self-repair. Spine (Phila Pa 1976). 2016;41(2):97-103.

13. Liu NK, et al. Altered microRNA expression following traumatic spinal cord injury. Exp Neurol. 2009;219(2):424-9.

14. Li P, Teng ZQ, Liu CM. Extrinsic and intrinsic regulation of axon regeneration by microRNAs after spinal cord injury. Neural Plast. 2016;2016:1279051.

15. Tumahai P, et al. Vitreous microparticle shedding in retinal detachment: a prospective comparative study. Invest Ophthalmol Vis Sci. 2016;57(1):40-6. Tumahai, P. et al. showed vitreous EVs derived from photoreceptors were higher in the aqueous humor of patients after rhegmatogenous RD.

16. Ragusa M, et al. MicroRNAs in vitreus humor from patients with ocular diseases. Mol Vis. 2013;19:430-40.

17. Ragusa $\mathrm{M}$, et al. miRNA profiling in vitreous humor, vitreal exosomes and serum from uveal melanoma patients: pathological and diagnostic implications. Cancer Biol Ther. 2015;16(9): 1387-96.

18. Santangelo A, et al. Circulating microRNAs as emerging noninvasive biomarkers for gliomas. Ann Transl Med. 2017;5(13):277.

19. Tanaka Y, et al. Profiles of extracellular miRNAs in the aqueous humor of glaucoma patients assessed with a microarray system. Sci Rep. 2014;4:5089.

20. Dismuke WM, et al. Human aqueous humor exosomes. Exp Eye Res. 2015;132:73-7.

21. Lerner N, Avissar S, Beit-Yannai E. Extracellular vesicles mediate signaling between the aqueous humor producing and draining cells in the ocular system. PLoS One. 2017;12(2):e0171153. Nonpigmented ciliary epithelium cell (NPCE)-derived EVs accumulate in trabecular meshwork cells and effect Wnt signaling, a pathway involved in intraocular pressure regulation.

22. Dunmire JJ, et al. MicroRNA in aqueous humor from patients with cataract. Exp Eye Res. 2013;108:68-71.

23. Pachler $\mathrm{K}$, et al. A good manufacturing practice-grade standard protocol for exclusively human mesenchymal stromal cell-derived extracellular vesicles. Cytotherapy. 2017;19(4):458-72.

24. Lotvall J, et al. Minimal experimental requirements for definition of extracellular vesicles and their functions: a position statement from the International Society for Extracellular Vesicles. J Extracell Vesicles. 2014;3:26913. 
25. Ko J, Carpenter E, Issadore D. Detection and isolation of circulating exosomes and microvesicles for cancer monitoring and diagnostics using micro-/nano-based devices. Analyst. 2016;141(2):450-60.

26. Keerthikumar S, et al. ExoCarta: a web-based compendium of exosomal cargo. J Mol Biol. 2016;428(4):688-92. The ExoCarta database was developed.

27. Kang GY, et al. Exosomal proteins in the aqueous humor as novel biomarkers in patients with neovascular age-related macular degeneration. J Proteome Res. 2014;13(2):581-95.

28. Elshelmani H, Rani S. Exosomal microRNA discovery in agerelated macular degeneration. Methods Mol Biol. 2017;1509: 93-113.

29. $\mathrm{Hu} \mathrm{B}$, et al. Effect of extracellular vesicles on neural functional recovery and immunologic suppression after rat cerebral apoplexy. Cell Physiol Biochem. 2016;40(1-2):155-62.

30. Mead B, Tomarev S. Bone marrow-derived mesenchymal stem cells-derived exosomes promote survival of retinal ganglion cells through miRNA-dependent mechanisms. Stem Cells Transl Med. 2017;6(4):1273-85. Mead et al. showed intravitreally injected EVs delivered their cargoes to both Müller glia and RGCs and increased RGC survival and axon regeneration after optic nerve ischemia.

31. Tassew NG, et al. Exosomes mediate mobilization of autocrine Wnt10b to promote axonal regeneration in the injured CNS. Cell Rep. 2017;20(1):99-111. Fibroblast EVs were reported to increase RGC survival and growth after acute optic nerve ischemia via established axon growth regulatory pathways involving mTOR activation by GSK3 $\beta$ and TSC2.

32. Moisseiev E, et al. Protective effect of intravitreal administration of exosomes derived from mesenchymal stem cells on retinal ischemia. Curr Eye Res. 2017:1-10.

33. Jaimes Y, et al. Mesenchymal stem cell-derived microvesicles modulate lipopolysaccharides-induced inflammatory responses to microglia cells. Stem Cells. 2017;35(3):812-23.

34. Liddelow SA, et al. Neurotoxic reactive astrocytes are induced by activated microglia. Nature. 2017;541(7638):481-7.

35. Yu B, et al. Exosomes derived from MSCs ameliorate retinal laser injury partially by inhibition of MCP-1. Sci Rep. 2016;6:34562. EVs were reported to reduce monocyte chemotactic protein (MCP)-1 expression and injury, apoptosis, and inflammatory responses in a mouse model of laser induced retinopathy.

36. van der Merwe Y, Steketee MB. Immunomodulatory approaches to CNS injury: extracellular matrix and exosomes from extracellular matrix conditioned macrophages. Neural Regen Res. 2016;11(4): 554-6.

37. Wassmer SJ, et al. Exosome-associated AAV2 vector mediates robust gene delivery into the murine retina upon intravitreal injection. Sci Rep. 2017;7:45329.

38. Kordelas L, et al. MSC-derived exosomes: a novel tool to treat therapy-refractory graft-versus-host disease. Leukemia. 2014;28(4):970-3. 